\title{
Hybrid stars within a covariant, nonlocal chiral quark model
}

\author{
D. B. Blaschke, ${ }^{1,2,3,{ }^{*}}$ D. Gómez Dumm, ${ }^{4,5, \dagger}$ A. G. Grunfeld, ${ }^{5,6, \ddagger}$ T. Klähn,,${ }^{3,7,8, \S}$ and N. N. Scoccola ${ }^{5,6,9, \|}$ \\ ${ }^{1}$ Institute for Theoretical Physics, University of Wroclaw, Max Born place 9, PL-50204 Wroclaw, Poland \\ ${ }^{2}$ Bogoliubov Laboratory of Theoretical Physics, JINR Dubna, Joliot-Curie Street 6, RU-141980 Dubna, Russia \\ ${ }^{3}$ Institut für Physik, Universität Rostock, Universitätsplatz 3, D-18051 Rostock, Germany \\ ${ }^{4}$ Instituto de Física La Plata, CONICET-Departamento de Física, Universidad Nacional de La Plata, C.C. 67, 1900 La Plata, Argentina \\ ${ }^{5}$ CONICET, Rivadavia 1917, 1033 Buenos Aires, Argentina \\ ${ }^{6}$ Physics Department, Comisión Nacional de Energía Atómica, Av. Libertador 8250, 1429 Buenos Aires, Argentina \\ ${ }^{7}$ Gesellschaft für Schwerionenforschung mbH (GSI), D-64291 Darmstadt, Germany \\ ${ }^{8}$ Physics Division, Argonne National Laboratory, Argonne, Illinois 60439-4843, USA \\ ${ }^{9}$ Universidad Favaloro, Solís 453, 1078 Buenos Aires, Argentina
}

(Received 17 April 2007; published 14 June 2007)

\begin{abstract}
We present a hybrid equation of state $(\mathrm{EOS})$ for dense matter in which a nuclear matter phase is described within the Dirac-Brueckner-Hartree-Fock (DBHF) approach and a two-flavor quark matter phase is modelled according to a recently developed covariant, nonlocal chiral quark model. We show that modern observational constraints for compact star masses $\left(M \sim 2 M_{\odot}\right)$ can be satisfied when a small vector-like four quark interaction is taken into account. The corresponding isospin symmetric EoS is consistent with flow data analyses of heavy ion collisions and points to a deconfinement transition at about $0.55 \mathrm{fm}^{-3}$.
\end{abstract}

DOI: 10.1103/PhysRevC.75.065804

PACS number(s): 04.40.Dg, 12.38.Mh, 26.60.+c, 97.60.Jd

\section{INTRODUCTION}

Understanding the properties of matter at moderate and high densities is required, e.g., to explain the astrophysical phenomena which accompany the birth of compact stars in supernova explosions, and the further evolution processes of cooling, spin-down, accretion, merging with companion stars, etc., which lead to effects accessible to observation.

Nowadays, one of the questions in the focus of discussions is the possibility of a phase transition to deconfined quark matter in the stellar cores. There is no doubt that deconfinement of quarks shall occur at sufficiently high densities, in accordance with the asymptotic freedom of QCD [1]. However, it is not clear a priori whether the critical density for deconfinement is low enough to be reached in the cores of neutron stars [2]. Even if this condition can be fulfilled, the properties of dense quark matter in the vicinity of the deconfinement transition might be too similar to those of dense hadronic matter to result in clearly distinguishable signals. On the other hand, there have been a number of interesting suggestions about how the occurrence of quark matter in neutron stars could manifest itself and possibly contribute to the resolution of puzzling observations. For the sake of illustration we remind the reader of the timing behavior of pulsar spin-down [3], frequency clustering [4], or population clustering [5,6] of accreting compact stars, which are based on a softening of the equation of state and therefore a compactification as well as a reduction of the maximum allowable stellar masses. Since deconfined quark

\footnotetext{
*blaschke@ift.uni.wroc.pl

†dumm@fisica.unlp.edu.ar

†ag.grunfeld@gmail.com

§thomas.klaehn@googlemail.com

"scoccola@tandar.cnea.gov.ar
}

matter is rather stiff when compared to hyperonic matter, the problem with rather low maximum masses of compact stars with hyperonic interior could be solved by the occurrence of a quark matter core [7]. Similarly, the description of the compact star cooling evolution with superconducting quark matter interior seems favorable over a purely hadronic modeling [8]. A deconfinement transition during the protoneutron star (PNS) evolution offers a mechanism to explain the gammaray burst energy release of the order of 100 bethe $\left(=10^{53}\right.$ erg) [9-11]. The nucleation timescales for a quark matter phase transition could explain the time delay statistics of gamma-ray burst (GRB) subpulse structure $[12,13]$. In the presence of a strong magnetic field, neutrino propagation in hot, superconducting quark matter can become collimated (beaming) and asymmetric, thus explaining a resulting kick velocity for the PNS [14].

The recent progress in compact star observations justifies a reinvestigation of the issue of hybrid stars with quark matter cores and theoretical aspects of dense matter properties. In particular, the high mass of $M=2.1 \pm 0.2 M_{\odot}$ for the pulsar J0751+1807 in a neutron star-white dwarf binary system [15] and the large radius of $R>12 \mathrm{~km}$ for the isolated neutron star RX J1856.5-3754 (shorthand: RX J1856) [16] point to a stiff equation of state at high densities. Measurements of high masses are also reported for compact stars in low-mass $\mathrm{X}$-ray binaries (LMXBs) as, e.g., $M=2.0 \pm 0.1 M_{\odot}$ for the compact object in $4 \mathrm{U}$ 1636-536 [17]. With data of this kind, new stringent constraints on the equation of state of strongly interacting matter at high densities have been formulated (see [18] and references therein). It has been argued [16,19] that deconfined quark matter cannot exist in the centers of compact stars with masses and radii as reported for these objects. In view of recent works on the quark matter EoS, however, this claim appears to be premature [20]. It has been demonstrated within the Nambu-Jona-Lasinio model for quark matter [21] 
that the inclusion of a diquark condensate (leading to color superconductivity) together with a vector meson condensate, not only provides a more elaborate description of the EoS but also allows us to describe the phenomenology of hybrid stars in excellent accordance with the above-mentioned new mass and mass-radius constraints. The two mechanisms at work are (1) the lowering of the phase transition density due to the diquark condensate, so that already typical neutron stars with masses in the range 1.1-1.5 $M_{\odot}$ can be hybrid stars with extended quark matter cores; (2) the stiffening of the EoS due to the vector mean field, which implies an increase of the maximum accessible masses of star configurations up to $\sim 2 M_{\odot}$.

Another lesson to be learned from NJL model studies is that at low temperatures there is a sequential deconfinement: strange quarks occur only at densities well above the deconfinement of light quarks [22-27]. If those densities could be reached in a compact star, the corresponding strange quark matter cores would be in a superconducting CFL phase, which renders the hybrid star configuration mechanically unstable $[21,28,29]$. In the present work we will restrict ourselves to the discussion of the two-flavor case, applying the more elaborate formalism of a recently developed nonlocal, covariant chiral quark model [30-32]. Moreover, we consider a generalized version of this model, including an isoscalar vector meson current which, in the same way as in the case of the Walecka model for nuclear matter, leads to a stiffening of the quark matter EoS.

Although we understand hadrons as bound states of quarks, there does not yet exist a unified approach which accurately describes the thermodynamics of the transition from nuclear to quark matter. Therefore, we apply a two-phase description by performing a Maxwell construction describing the transition from a nuclear matter EoS to the quark matter EoS. The nuclear equation of state to be considered here results from calculations within the DBHF approach [33] and has already been applied for the description of compact stars before [18,21]. Since this EoS is rather stiff, several modern compact star observations have been well reproduced. In particular, within this approach one is able to obtain large neutron star masses $\left(M_{\max }=\right.$ $\left.2.33 M_{\odot}\right)$ and radii $(R=12-13 \mathrm{~km}$ for typical neutron stars). On the other hand, the DBHF description is not so well suited to reproduce the results obtained from elliptic flow data in symmetric nuclear matter (SNM): from these results, it can be seen that the DBHF EoS tends to be too stiff beyond densities of 3 times the saturation density $n_{\text {sat }}=0.16 \mathrm{fm}^{-3}$ [18]. We show here that this problem can be solved by a phase transition to quark matter while simultaneously fulfilling the constraints on the behavior of dense matter for the case of hybrid stars.

The article is organized as follows. A brief description of the DBHF approach and the quark matter model used here is given in Secs. II A and II B, respectively. In Sec. III we show our numerical results, comparing them with present empirical constraints on the behavior of dense matter under constraints for conditions in neutron stars and heavy ion collisions. Our conclusions are stated in Sec. IV. Finally, in the Appendix we provide some details of the quark matter model.

\section{THEORETICAL FORMALISM}

As mentioned in the Introduction, in the present calculation we use a two phase description to account for the transition from a nuclear matter EoS to a quark matter EoS. In the following two subsections we briefly discuss the theoretical approaches considered here to describe each of these phases.

\section{A. Nuclear matter equation of state}

For the description of the nuclear matter equation of state we rely on the relativistic Dirac-Brueckner-Hartree-Fock (DBHF) approach where the nucleon inside the medium is dressed by the self-energy $\Sigma$. This self-energy is obtained from the Bethe-Salpeter equation for the nucleon-nucleon T-matrix in the ladder approximation, using the Bonn-A potential in the interaction kernel [34].

We employ a parametrization of the resulting EoS based on a parabolic dependence of the energy per nucleon on the asymmetry parameter $\alpha=1-2 x$, given in the form

$$
E(n, \alpha)=E_{0}(n)+\alpha^{2} E_{S}(n),
$$

where $x=n_{p} / n$ is the proton fraction, $E_{0}(n)$ is the energy per nucleon in SNM, and $E_{S}(n)$ is the (a)symmetry energy. Both contributions $E_{0}(n)$ and $E_{S}(n)$ have been extracted from DBHF calculations for the cases $\alpha=0$ and $\alpha=1$, respectively. The parabolic interpolation in Eq. (1) has been widely used in the literature, see, e.g., Refs. [18,35], and proves to be an excellent parametrization of the asymmetry dependence for the purpose of the present study. An exact reproduction of a given EoS might require higher order terms, which have been neglected here. The advantage of the parabolic interpolation lies in the fact that all zero temperature equations of state for neutron star matter (NSM) can be derived by applying simple thermodynamic identities [36]. In particular, we obtain

$$
\begin{aligned}
\varepsilon_{B}(n, \alpha)= & n E(n, \alpha), \\
P_{B}(n, \alpha)= & n^{2} \frac{\partial}{\partial n} E(n, \alpha), \\
\mu_{n, p}(n, \alpha)= & \left(1+n \frac{\partial}{\partial n}\right) E_{0}(n) \\
& -\left(\alpha^{2} \mp 2 \alpha-\alpha^{2} n \frac{\partial}{\partial n}\right) E_{S}(n),
\end{aligned}
$$

for the baryonic energy density $\varepsilon(n)$, the pressure $P(n)$, and the chemical potentials of neutrons $\mu_{n}$ (upper sign) and protons $\mu_{p}$ (lower sign), respectively.

\section{B. Quark matter equation of state}

Early reviews on the treatment of quark matter within the NJL model as a chiral quark model with a local currentcurrent-type interaction can be found, e.g., in Refs. [37-39]. In order to include color superconductivity, techniques were needed which are described, e.g., in Refs. [40,41].

We use here a generalization of these approaches to a nonlocal chiral quark model which includes scalar and vector 
quark-antiquark interactions and antitriplet scalar diquark interactions. The corresponding effective Euclidean action in the case of two light flavors is given by

$$
\begin{aligned}
S_{E}= & \int d^{4} x\left\{\bar{\psi}(x)\left(-i \gamma^{\mu} \partial_{\mu}+m_{c}\right) \psi(x)-\frac{G_{S}}{2} j_{S}^{f}(x) j_{S}^{f}(x)\right. \\
& \left.-\frac{H}{2}\left[j_{D}^{a}(x)\right]^{\dagger} j_{D}^{a}(x)-\frac{G_{V}}{2} j_{V}^{\mu}(x) j_{V}^{\mu}(x)\right\}
\end{aligned}
$$

Here $m_{c}$ is the current quark mass, which is assumed to be equal for $u$ and $d$ quarks, whereas the currents $j_{S, D, V}(x)$ are given by nonlocal operators based on a separable approximation to the effective one gluon exchange model (OGE) of QCD. These currents read

$$
\begin{aligned}
& j_{S}^{f}(x)=\int d^{4} z g(z) \bar{\psi}\left(x+\frac{z}{2}\right) \Gamma_{f} \psi\left(x-\frac{z}{2}\right), \\
& j_{D}^{a}(x)=\int d^{4} z g(z) \bar{\psi}_{C}\left(x+\frac{z}{2}\right) i \gamma_{5} \tau_{2} \lambda_{a} \psi\left(x-\frac{z}{2}\right), \\
& j_{V}^{\mu}(x)=\int d^{4} z g(z) \bar{\psi}\left(x+\frac{z}{2}\right) \gamma_{\mu} \psi\left(x-\frac{z}{2}\right),
\end{aligned}
$$

where we have defined $\psi_{C}(x)=\gamma_{2} \gamma_{4} \bar{\psi}^{T}(x)$ and $\Gamma_{f}=$ (11, $\left.i \gamma_{5} \vec{\tau}\right)$, while $\vec{\tau}$ and $\lambda_{a}$, with $a=2,5,7$, stand for Pauli and Gell-Mann matrices acting on flavor and color spaces, respectively [notice that $\gamma_{\mu}=\left(\vec{\gamma}, \gamma_{4}\right)$ are Euclidean Dirac matrices]. The functions $g(z)$ in Eqs. (7) are nonlocal covariant form factors characterizing the effective quark interaction [32].

The effective action in Eq. (5) might arise via Fierz rearrangement from some underlying more fundamental interactions, and is understood to be used-at the mean field level -in the Hartree approximation. In general, the ratios of coupling constants $H / G_{S}, G_{V} / G_{S}$ would be determined by this microscopic couplings; for example, OGE interactions in the vacuum lead to $H / G_{S}=0.75$ and $G_{V} / G_{S}=0.5$. However, since the precise derivation of effective couplings from QCD is not known, there is a large theoretical uncertainty in these ratios. Details of the values used in the present work will be given below.

We proceed by considering a bosonized version of this quark model, in which scalar, vector, and diquark fields are introduced. Moreover, we expand these fields around their respective mean field values, keeping the lowest order contribution to the thermodynamic quantities. The only nonvanishing mean field values in the scalar and vector sectors correspond to isospin zero fields, $\bar{\sigma}$ and $\bar{\omega}$, respectively, while in the diquark sector, owing to the color symmetry, one can rotate in color space to fix $\bar{\Delta}_{5}=\bar{\Delta}_{7}=0, \bar{\Delta}_{2}=\bar{\Delta}$.

Now we consider the Euclidean action at zero temperature and finite baryon chemical potential $\mu_{B}$. Introducing different chemical potentials $\mu_{f c}$ for each flavor and color, the corresponding mean field grand canonical thermodynamic potential per unit volume can be written as

$$
\begin{aligned}
\Omega^{\mathrm{MFA}}= & \frac{\bar{\sigma}^{2}}{2 G_{S}}+\frac{\bar{\Delta}^{2}}{2 H}-\frac{\bar{\omega}^{2}}{2 G_{V}} \\
& -\frac{1}{2} \int \frac{d^{4} p}{(2 \pi)^{4}} \ln \operatorname{det}\left[S^{-1}\left(\bar{\sigma}, \bar{\Delta}, \bar{\omega}, \mu_{f c}\right)\right],
\end{aligned}
$$

where the inverse propagator $S^{-1}$ is a $48 \times 48$ matrix in Dirac, flavor, color, and Nambu-Gorkov spaces [30,31]. Its explicit regularized form $\Omega_{(\mathrm{reg})}^{\mathrm{MFA}}$ is given in the Appendix, together with further details about the model. The mean field values $\bar{\sigma}, \bar{\Delta}$ and $\bar{\omega}$ are obtained from the coupled equations

$$
\frac{d \Omega^{\mathrm{MFA}}}{d \bar{\Delta}}=0, \quad \frac{d \Omega^{\mathrm{MFA}}}{d \bar{\sigma}}=0, \quad \frac{d \Omega^{\mathrm{MFA}}}{d \bar{\omega}}=0 .
$$

In principle one has six different quark chemical potentials, corresponding to quark flavors $u$ and $d$ and quark colors $r, g$, and $b$. However, there is a residual color symmetry (say, between red and green colors) arising from the direction of $\bar{\Delta}$ in color space. Moreover, if we require the system to be in chemical equilibrium, it can be seen that chemical potentials are not independent from each other. In general, it is shown that all $\mu_{f c}$ can be written in terms of three independent quantities: the baryonic chemical potential $\mu_{B}$, a quark electric chemical potential $\mu_{Q_{q}}$ and a color chemical potential $\mu_{8}$. The corresponding relations read

$$
\begin{aligned}
& \mu_{u r}=\mu_{u g}=\frac{\mu_{B}}{3}+\frac{2}{3} \mu_{Q_{q}}+\frac{1}{3} \mu_{8}, \\
& \mu_{u b}=\frac{\mu_{B}}{3}+\frac{2}{3} \mu_{Q_{q}}-\frac{2}{3} \mu_{8}, \\
& \mu_{d r}=\mu_{d g}=\frac{\mu_{B}}{3}-\frac{1}{3} \mu_{Q_{q}}+\frac{1}{3} \mu_{8}, \\
& \mu_{d b}=\frac{\mu_{B}}{3}-\frac{1}{3} \mu_{Q_{q}}-\frac{2}{3} \mu_{8} .
\end{aligned}
$$

The chemical potential $\mu_{Q_{q}}$, which distinguishes between up and down quarks, as well as the color chemical potential $\mu_{8}$, which has to be introduced to ensure color neutrality [42-44], vanish for an isospin symmetric quark matter system. Thus, in this case, the corresponding EoS can be obtained after calculating the mean field values $\bar{\sigma}, \bar{\omega}$ and $\bar{\Delta}$ from Eqs. (8).

Now, if we want to describe the behavior of quark matter in the core of neutron stars, in addition to quark matter we have to take into account the presence of electrons and muons. Thus, treating leptons as a free relativistic Fermi gas, the total pressure of the quark matter + lepton system is given by

$$
P=-\Omega_{(\mathrm{reg})}^{\mathrm{MFA}}-\Omega^{l},
$$

where $\Omega^{l}$ is the thermodynamical potential per unit volume for a gas of noninteracting electrons and muons (see Appendix). In addition, it is necessary to take into account that quark matter has to be in $\beta$ equilibrium with electrons and muons through the $\beta$ decay reactions

$$
d \rightarrow u+l+\bar{v}_{l}, \quad u+l \rightarrow d+v_{l},
$$

for $l=e, \mu$. Thus, assuming that (anti)neutrinos escape from the stellar core, we have an additional relation between fermion chemical potentials, namely,

$$
\mu_{d c}-\mu_{u c}=-\mu_{Q_{q}}=\mu_{l}
$$

for $c=r, g, b, \mu_{e}=\mu_{\mu}=\mu_{l}$.

Finally, in the core of neutron stars we also require the system to be electric and color charge neutral, hence the number of independent chemical potentials reduces further. 



FIG. 1. (Color online) Pressure as a function of the baryochemical potential for isospin symmetric matter. The nuclear matter phase is modelled by the DBHF equation of state with the Bonn-A potential [33] (solid line) and the results for the covariant, nonlocal chiral quark model are given for different scaled vector coupling strengths $g=0.03,0.05,0.07,0.09$ (dotted, dash-dotted, dashed, and dash-doubledotted curves, respectively) and scaled diquark coupling strengths of $h=0.70$ (left panel) and $h=0.74$ (right panel). A phase transition to quark matter is obtained at the crossing of nuclear and quark matter curves, for discussion see text.
Indeed, $\mu_{l}$ and $\mu_{8}$ get fixed by the condition that charge and color densities vanish,

$$
\begin{aligned}
\rho_{Q_{t o t}} & =\rho_{Q_{q}}-\sum_{l=e, \mu} \rho_{l} \\
& =\sum_{c=r, g, b}\left(\frac{2}{3} \rho_{u c}-\frac{1}{3} \rho_{d c}\right)-\sum_{l=e, \mu} \rho_{l}=0, \\
\rho_{8} & =\frac{1}{\sqrt{3}} \sum_{f=u, d}\left(\rho_{f r}+\rho_{f g}-2 \rho_{f b}\right)=0,
\end{aligned}
$$

where the expressions for the lepton densities $\rho_{l}$ and the quark densities $\rho_{f c}$ can be found in the Appendix. Note that the set of color chemical potentials required to ensure color neutrality of the system depends on the choice of the orientation of the diquark condensate orientation in color space [45]. For the standard choice employed in the present work, $\mu_{8}$ is sufficient. In summary, in the case of neutron star quark matter, for each value of $\mu_{B}$ one can find the values of $\bar{\Delta}, \bar{\sigma}, \omega, \mu_{l}$, and $\mu_{8}$ by solving Eqs. (8), supplemented by Eqs. (12) and (13). This allows us to obtain the quark matter EoS in the thermodynamic region we are interested in.

\section{NUMERICAL RESULTS AND DISCUSSION}

In this section we present our numerical results, showing the behavior of both isospin symmetric hadronic matter and neutral hadronic matter for finite baryochemical potential. The nuclear-to-quark matter phase transition is treated in the traditional way, following a two-phase scheme in which the nuclear and quark matter phases are described by the theoretical approaches presented in the previous section.

As stated, the nuclear matter phase is described according to the DBHF approach, in which the nucleon self-energy is calculated from the Bethe-Salpeter equation considering a Bonn-A potential in the interaction kernel. Regarding the quark matter model, we note first that, according to previous analyses carried out within nonlocal scenarios [30,31], the results are not expected to show a strong qualitative dependence on the shape of the nonlocal form factors. Thus we will consider (in momentum space) a simple and well-behaved Gaussian function,

$$
g\left(p^{2}\right)=\exp \left(-p^{2} / \Lambda^{2}\right)
$$

where $\Lambda$ is a free parameter of the model, playing the role of an ultraviolet cutoff. The value of $\Lambda$, as well as the values of the free model parameters $G_{S}$ and $m_{c}$, can be fixed from low energy phenomenology. Here we have chosen these input parameters so as to reproduce the empirical values for the pion mass $m_{\pi}=139 \mathrm{MeV}$ and decay constant $f_{\pi}=92.4 \mathrm{MeV}$, and to obtain a phenomenologically reasonable value for the chiral condensate at vanishing $\mu_{B}$, namely, $\langle 0|\bar{q} q| 0\rangle^{1 / 3}=-230 \mathrm{MeV}$. In this way we obtain $m_{c}=6.49 \mathrm{MeV}, G_{S}=0.515 \times$ $10^{-4} \mathrm{MeV}^{-2}$, and $\Lambda=678 \mathrm{MeV}$ [46].

The values of the scalar diquark coupling and the isoscalar vector coupling $H$ and $G_{V}$, or equivalently the dimensionless ratios $h=H / G_{S}$ and $g=G_{V} / G_{S}$, are considered here as parameters to be chosen in accordance with phenomenological constraints from flow data analyses of heavy-ion collisions and the new mass and mass-radius constraints from compact star observations. In accordance with our previous investigation of the phase diagram of neutral quark matter in Ref. [32] we choose two typical values $h=0.70$ and $h=0.74$ for the diquark/scalar coupling ratio, and analyze the phase transition features for different values of $g$ so as to obtain an acceptable transition density.

In Fig. 1 we show the curves for the pressure as function of the baryochemical potential, for both nuclear and quark matter phases, in the case of isospin symmetric matter. Left and right panels correspond to $h=0.70$ and $h=0.74$, respectively, and in each case three values of the scaled vector coupling $g$ have been chosen. As can be seen from the figures, within these ranges of $g$ both nuclear and quark matter EoS behave similarly in the relevant domain of baryochemical potentials, therefore the value of the critical $\mu_{B}$ strongly depends on the value of the vector coupling. In any case, for both $h=0.70$ and $h=0.74$ it is possible to tune the value of $g$ so that the softening due to the deconfinement transition occurs at a density of about $0.55 \mathrm{fm}^{-3}$. At this point the DBHF EoS becomes too stiff, being unable to fulfill the flow constraint [47]. This situation is sketched in Fig. 2, where we show the area allowed by the flow constraint and the corresponding DBHF curve in the density-pressure plane. For $h=0.70$ and $h=0.74$, the values $g=0.05$ and $g=0.07$, respectively (dotted lines in the $P-\mu_{B}$ curves of Fig. 1) lead to nuclear-to-quark matter phase transitions such that the stiffness of the pressure curve is softened and the flow constraint can be satisfied. On the other hand, values of $g$ outside the range considered in Fig. 1 would 




FIG. 2. (Color online) Pressure as a function of the density for isospin symmetric matter. The phase transition to quark matter softens the EoS at densities above $0.55 \mathrm{fm}^{-3}$, relative to the nuclear DBHF EoS, thus allowing us to fulfill the flow constraint derived in Ref. [47]. Line styles as in Fig. 1.

lead to either a too early phase transition or to a situation in which there is no transition to quark matter at all. We emphasize that both the nuclear and quark matter EsoS behave almost identically over a wide range of the baryochemical potential in Fig. 1. This results in an almost direct crossover transition with a small or even negligible density jump at the transition density, as shown in Fig. 2. As a further consequence, the transition density strongly depends on small changes of $g$.

Finally, let us take into account the hadronic matter models leading to the curves in Fig. 2, considering now the description of neutral hadronic matter in the interior of compact stars. In Fig. 3, we show the sequences of compact star configurations obtained as solutions of the Tolman-Oppenheimer-Volkoff equations of general-relativistic hydrodynamic stability, for self-gravitating dense hadronic matter described by these theoretical models. The results quoted in Fig. 3 represent the main outcome of this work: using the quark matter EoS derived from a covariant nonlocal chiral quark model, generalized here by including a vector interaction, we obtain hybrid star configurations which fulfill the modern constraints on high masses and radii of compact stars discussed in the Introduction.



FIG. 3. (Color online) Mass-radius relationships for neutron star configurations (DBHF EoS, solid line) and hybrid star configurations with hadronic shell (DBHF EoS) and color superconducting quark matter core (nonlocal chiral quark model EoS) for two parameter sets characterized by the coupling ratios $h=H / G_{S}$ and $g=G_{V} / G_{S}$. Dashed line: $h=0.74, g=0.07$; dash-dotted line: $h=0.70, g=$ 0.05 .
Moreover, the deconfinement transition for isospin symmetric matter occurs at about threefold nuclear saturation density, and results in a sufficient softening of the EoS thus circumventing a violation of the flow constraint from heavy-ion collisions.

\section{CONCLUSIONS}

We have generalized in this work a recently developed covariant, nonlocal chiral quark model by including a vectorvector four quark interaction which leads to a stiffening of the corresponding quark matter EoS. This allows us to describe compact stars possessing a quark matter core and being in agreement with the modern compact star phenomenology, which suggests stars with maximum masses of $\sim 2 M_{\odot}$ and radii of $12-13 \mathrm{~km}$.

We show that the traditional application of Gibbs conditions for phase equilibrium to construct a phase transition between deconfined quark matter-described in the new approach developed here - and nuclear matter - described by the DBHF approach — with the Bonn-A potential, is very sensitive to tiny changes of parameter values: quark and hadronic matter EoS's behave rather similarly in the vicinity of the phase transition and lead to a crossover-like behavior for the hybrid EoS. This reconfirms an earlier discussed 'masquerade' effect $[8,21,48]$ for hybrid stars within the present approach. After exploring the allowed range of model parameters, we find values for which both the flow constraint from heavy-ion collisions and the mass constraint for hybrid stars are satisfied.

We want to point out that the deconfinement phase transition should be a rather robust phenomenon of the high-density EoS. Therefore, the mentioned 'masquerade' effect may point to a deficiency in the two phase approach for the description of hybrid EoS's. It is a demanding task to develop unified approaches for quark/nuclear matter on the basis of chiral quark models in which nucleons and mesons appear as relativistic bound states of quarks and antiquarks (for first steps in this direction, see Refs. [49,50]). Under conditions of high density and/or temperature these bound states dissociate into continuum correlations (resonances) in quark matter within a Mott transition (see [51] for a model calculation within a nonrelativistic Green functions approach, which has also lead to an early prediction of stable quark matter cores in compact stars [52]).

We believe that the covariant nonlocal chiral quark model presented here could be developed to a more elaborate approach unifying quark and hadronic matter descriptions on the quark level. The realization of such a project is beyond the scope of the present work.

\section{ACKNOWLEDGMENTS}

This work has been supported in part by CONICET and ANPCyT (Argentina), under grants PIP 6009, PIP 6084, and PICT04-03-25374, and by a scientist exchange program between Germany and Argentina funded jointly by DAAD and ANTORCHAS under grant Nos. DE/04/27956 and 4248-6, respectively. T.K. acknowledges support by the GSI Darmstadt 
and D.B.B. by the Polish Ministry of Science and Higher Education.

\section{APPENDIX: DETAILS OF THE NONLOCAL MODEL FOR QUARK MATTER}

In this appendix we show some explicit expressions corresponding to the nonlocal chiral quark model considered in Sec. II B. The determinant of the $48 \times 48$ matrix $S^{-1}$ appearing in Eq. (7) can be analytically calculated. In this way one obtains

$$
\Omega^{\mathrm{MFA}}=\frac{\bar{\sigma}^{2}}{2 G_{S}}+\frac{\bar{\Delta}^{2}}{2 H}-\frac{\bar{\omega}^{2}}{2 G_{V}}-\int \frac{d^{4} p}{(2 \pi)^{4}} \sum_{c=r, g, b} \ln \left|A_{c}\right|^{2},
$$

where

$$
\begin{aligned}
A_{c}= & {\left[\left(\tilde{p}_{u c}^{+}\right)^{2}+\left(\Sigma_{u c}^{p}\right)^{2}\right]\left[\left(\tilde{p}_{d c}^{-}\right)^{2}+\left(\Sigma_{d c}^{p *}\right)^{2}\right] } \\
& +\left(1-\delta_{b c}\right) \Delta^{p^{2}}\left[\Delta^{p^{2}}+2 \tilde{p}_{u c}^{+} \cdot \tilde{p}_{d c}^{-}+2 \Sigma_{u c}^{p} \Sigma_{d c}^{p *}\right],
\end{aligned}
$$

with the following definitions:

$$
\begin{aligned}
\tilde{p}_{f c}^{ \pm} & =\left(\vec{p}, p_{4} \mp i\left[\mu_{f c}-\bar{\omega} g\left(p_{f c}^{ \pm 2}\right)\right]\right), \\
p_{f c}^{ \pm} & =\left(\vec{p}, p_{4} \mp i \mu_{f c}\right), \\
\Sigma_{f c}^{p} & =m_{f}+\bar{\sigma} g\left(p_{f c}^{+2}\right) \\
\Delta^{p} & =\bar{\Delta} g\left(\frac{\left[p_{u r}^{+}+p_{d r}^{-}\right]^{2}}{4}\right),
\end{aligned}
$$

where $f=u, d$, and $c=r, g, b$. Notice that due to the symmetry between red and green colors one has $\mu_{f r}=$ $\mu_{f g}$.

In general, for finite values of the current quark mass, $\Omega^{\mathrm{MFA}}$ turns out to be divergent. We have used here a regularization procedure in which we add and subtract the thermodynamical potential for a free quark gas, namely,

$$
\Omega_{(\text {reg })}^{\mathrm{MFA}}=\Omega^{\mathrm{MFA}}-\Omega_{(\text {nonreg })}^{\text {free }}+\sum_{f, c} \Omega_{(\mathrm{reg}) f c}^{\mathrm{free}},
$$

In the right hand side, $\Omega_{\text {(nonreg) }}^{\text {free }}$ is obtained from Eq. (A1) just by setting $\bar{\Delta}=\bar{\sigma}=\bar{\omega}=0$, while for each fermion species $i$ the regularized free thermodynamical potential $\Omega_{(\mathrm{reg}) i}^{\text {free }}$ is given by

$$
\Omega_{(\mathrm{reg}) i}^{\mathrm{free}}=-\frac{1}{24 \pi^{2}} m_{i}^{4} F\left(\mu_{i} / m_{i}\right)
$$

with

$$
F(x)=2 x\left(x^{2}-5 / 2\right) \sqrt{x^{2}-1}+3 \ln \left(x+\sqrt{x^{2}-1}\right) .
$$

The sum in Eq. (A5) extends over all quark flavors and colors. In the case of leptons, since they can be treated as free particles, the corresponding contribution to the compact star thermodynamical potential is simply given by

$$
\Omega^{l}=\sum_{l} \Omega_{(\mathrm{reg}) l}^{\mathrm{free}},
$$

with $l=e, \mu$.

Finally, according to our regularization prescription, the fermion densities appearing in Eq. (13) are given by

$$
\begin{aligned}
\rho_{f c} & =-\frac{\partial \Omega^{\mathrm{MFA}}}{\partial \mu_{f c}}-\frac{\partial \Omega_{(\text {(nonreg) }}^{\text {free }}}{\partial \mu_{f c}}+\rho_{(\mathrm{reg})}^{\text {free }} f c \\
\rho_{l} & =\rho_{(\mathrm{reg})}^{\text {free }},
\end{aligned}
$$

where $f=u, d, c=r, g, b$, and $l=e, \mu$. The fermion density $\rho_{(\mathrm{reg})_{i}}^{\text {free }}$ of a free particle gas can be easily obtained from the regularized thermodynamical potential in Eq. (A6), yielding

$$
\rho_{(\text {reg })_{i}}^{\text {free }}=\frac{1}{3 \pi^{2}}\left(\mu_{i}^{2}-m_{i}^{2}\right)^{3 / 2}
$$

while explicit expressions for the partial derivatives in Eq. (A9) can be obtained from the results quoted in Ref. [32], taking the $T=0$ limit.
[1] J. C. Collins and M. J. Perry, Phys. Rev. Lett. 34, 1353 (1975).

[2] G. Baym and S. A. Chin, Phys. Lett. B62, 241 (1976).

[3] N. K. Glendenning, S. Pei, and F. Weber, Phys. Rev. Lett. 79, 1603 (1997).

[4] N. K. Glendenning and F. Weber, Astrophys. J. Lett. 559, L119 (2001).

[5] G. S. Poghosyan, H. Grigorian, and D. Blaschke, Astrophys. J. Lett. 551, L73 (2001).

[6] D. Blaschke, I. Bombaci, H. Grigorian, and G. S. Poghosyan, New Astron. 7, 107 (2002).

[7] M. Baldo, G. F. Burgio, and H. J. Schulze, in Superdense QCD Matter and Compact Stars, NATO Science Series II (Springer, Dordrecht, 2006), Vol. 197, p. 113.

[8] D. Blaschke and H. Grigorian, Prog. Part. Nucl. Phys. 59, 139 (2007).

[9] I. Bombaci and B. Datta, Astrophys. J. Lett. 530, L69 (2000).

[10] Z. Berezhiani, I. Bombaci, A. Drago, F. Frontera, and A. Lavagno, Astrophys. J. 586, 1250 (2003).
[11] D. N. Aguilera, D. Blaschke, and H. Grigorian, Astron. Astrophys. 416, 991 (2004).

[12] A. Drago, G. Pagliara, and I. Parenti, Nucl. Phys. A782, 418 (2007).

[13] A. Drago, A. Lavagno, and G. Pagliara, Nucl. Phys. A774, 823 (2006).

[14] J. Berdermann, D. Blaschke, H. Grigorian, and D. N. Voskresensky, Prog. Part. Nucl. Phys. 57, 334 (2006).

[15] D. J. Nice, E. M. Splaver, I. H. Stairs, O. Löhmer, A. Jessner, M. Kramer, and J. M. Cordes, Astrophys. J. 634, 1242 (2005).

[16] J. E. Trümper, V. Burwitz, F. Haberl, and V. E. Zavlin, Nucl. Phys. B Proc. Sup. 132, 560 (2004).

[17] D. Barret, J. F. Olive, and M. C. Miller, Mon. Not. R. Astron. Soc. 361, 855 (2005).

[18] T. Klähn et al., Phys. Rev. C 74, 035802 (2006)

[19] F. Özel, Nat. Phys. 441, 1115 (2006).

[20] M. Alford, D. Blaschke, A. Drago, T. Klähn, G. Pagliara, and J. Schaffner-Bielich, Nat. Phys. 445, E7 (2007). 
[21] T. Klähn, D. Blaschke, F. Sandin, C. Fuchs, A. Faessler, H. Grigorian, G. Röpke, and J. Trümper, arXiv:nucl-th/0609067.

[22] C. Gocke, D. Blaschke, A. Khalatyan, and H. Grigorian, arXiv:hep-ph/0104183.

[23] S. B. Rüster, V. Werth, M. Buballa, I. A. Shovkovy, and D. H. Rischke, Phys. Rev. D 72, 034004 (2005).

[24] D. Blaschke, S. Fredriksson, H. Grigorian, A. M. Öztas, and F. Sandin, Phys. Rev. D 72, 065020 (2005).

[25] H. Abuki and T. Kunihiro, Nucl. Phys. A768, 118 (2006).

[26] H. J. Warringa, D. Boer, and J. O. Andersen, Phys. Rev. D 72, 014015 (2005).

[27] H. J. Warringa, arXiv:hep-ph/0606063.

[28] M. Baldo, M. Buballa, F. Burgio, F. Neumann, M. Oertel, and H. J. Schulze, Phys. Lett. B562, 153 (2003).

[29] M. Buballa, F. Neumann, M. Oertel, and I. Shovkovy, Phys. Lett. B595, 36 (2004).

[30] D. Gomez Dumm and N. N. Scoccola, Phys. Rev. D 65, 074021 (2002).

[31] R. S. Duhau, A. G. Grunfeld, and N. N. Scoccola, Phys. Rev. D 70, 074026 (2004).

[32] D. Gomez Dumm, D. B. Blaschke, A. G. Grunfeld, and N. N. Scoccola, Phys. Rev. D 73, 114019 (2006).

[33] E. N. E. van Dalen, C. Fuchs, and A. Faessler, Nucl. Phys. A744, 227 (2004).

[34] C. Fuchs, Springer Lect. Notes Phys. 641, 119 (2004).

[35] J. M. Lattimer and M. Prakash, Astrophys. J. 550, 426 (2001).
[36] M. Baldo, G. F. Burgio, and H. J. Schulze, Phys. Rev. C 61, 055801 (2000).

[37] U. Vogl and W. Weise, Prog. Part. Nucl. Phys. 27, 195 (1991).

[38] S. P. Klevansky, Rev. Mod. Phys. 64, 649 (1992).

[39] T. Hatsuda and T. Kunihiro, Phys. Rep. 247, 221 (1994).

[40] J. Berges and K. Rajagopal, Nucl. Phys. B538, 215 (1999).

[41] M. Buballa, Phys. Rep. 407, 205 (2005).

[42] K. Iida and G. Baym, Phys. Rev. D 63, 074018 (2001) [Erratumibid. 66, 059903 (2002)].

[43] M. Alford and K. Rajagopal, J. High Energy Phys. 06 (2002) 031.

[44] A. W. Steiner, S. Reddy, and M. Prakash, Phys. Rev. D 66, 094007 (2002).

[45] M. Buballa and I. A. Shovkovy, Phys. Rev. D 72, 097501 (2005).

[46] D. Gomez Dumm, A. G. Grunfeld, and N. N. Scoccola, Phys. Rev. D 74, 054026 (2006).

[47] P. Danielewicz, R. Lacey, and W. G. Lynch, Science 298, 1592 (2002).

[48] M. Alford, M. Braby, M. W. Paris, and S. Reddy, Astrophys. J. 629, 969 (2005).

[49] S. Lawley, W. Bentz, and A. W. Thomas, J. Phys. G 32, 667 (2006).

[50] A. H. Rezaeian and H. J. Pirner, Nucl. Phys. A779, 197 (2006).

[51] G. Röpke, D. Blaschke, and H. Schulz, Phys. Rev. D 34, 3499 (1986).

[52] D. Blaschke, T. Tovmasian, and B. Kämpfer, Sov. J. Nucl. Phys. 52, 675 (1990) [Yad. Fiz. 52, 1059 (1990)]. 